\title{
ЛАГЕРРОВЫ УРАВНЕНИЯ СОСТОЯНИЯ МНОГОМЕРНОЙ ДИСКРЕТНОЙ СИСТЕМЫ
}

\author{
(Представил Н. Алумяэ)
}

\section{1. Введение}

Теория линейных динамических систем, в том числе дискретных, весьма хорошо развита. Различные задачи анализа и синтеза систем управления не вызывают принципиальных трудностей и подробно исследованы как во временной, так и в частотной области. Во многих случаях успешно использованы представления входных и выходных процессов в ортогональном базисе, причем наиболее подходящим базисом для дискретных динамических систем признаны разностные степенные многочлены экспоненциального характера $\left[{ }^{1,2}\right]$.

Объектом внимания данной работы являются разностные многочлены типа Лагерра, введенные М. Готтлибом еще в 30 -е годы $\left[{ }^{3}\right]$. Эти многочлены обладают рядом положительных свойств: они удовлетворяют условию ортонормальности, легко вычисляются, имеют экспоненциальный характер, их свойства можно варьировать в широких пределах с помощью произвольно выбираемой постоянной. В теории линейных динамических систем они нашли пока ограниченное использование в задачах идентификации $\left[{ }^{4}\right]$ и фильтрации $\left[{ }^{2}\right]$.

В работе найдено новое представление дискретной динамической системы - лагерровы уравнения состояния, т. е. уравнения состояния между коэффициентами Фурье разложения входных и выходных процессов по разностным многочленам Лагерра, и получены простые формулы, обеспечивающие переход от обыкновенных уравнений состояния к лагерровым уравнениям состояния и наоборот. Показано, что система лагерровых уравнений состояния имеет те же качественные свойства (устойчивость, управляемость, наблюдаемость), что и исходная система. Поэтому, в принципе, лагерровы уравнения состояния могут быть использованы для решения различных задач анализа и синтеза систем управления.

Лагерровы уравнения состояния можно найти и по экспериментальным данным, в частности по переходным (или весовым) характеристикам системы.

Одна из возможностей лагеррового представления линейной динамической системы показана ниже на примере задачи построения эталонной модели системы управления по классическим показателям качества. 


\section{2. Разностные многочлены Лагерра}

Разностные многочлены Лагерра порядка $k$ определяются следующим образом $\left[{ }^{3}\right]$ :

$$
\gamma_{k}(t)=\mathrm{e}^{v t} \Delta^{k}\left[\left(\begin{array}{l}
t \\
k
\end{array}\right) \mathrm{e}^{-v t}\right], \quad t=0,1,2, \ldots,
$$

где $\Delta$ - оператор разностей, $v$ - положительное вещественное число, $\left(\begin{array}{l}t \\ k\end{array}\right)$ - биномиальный коэффициент. Нормированные многочлены [2]

$$
\psi_{k}(t)=(-1)^{k} \sqrt{\left(1-\xi^{2}\right) / \xi^{2 k-t}} \gamma_{k}(t),
$$

где $\xi=\sqrt{\mathrm{e}^{-v}}-$ постоянная Лагерра, можно привести к явному виду

$$
\psi_{k}(t)=\sqrt{1-\xi^{2}} \sum_{j=0}^{k}(-1)^{k+j}\left(\begin{array}{c}
k \\
j
\end{array}\right)\left(\begin{array}{c}
t+k-j \\
k
\end{array}\right) \xi^{t+k-2 j} .
$$

Вычисление многочленов $\psi_{k}(t)$ облегчают следующие рекуррентные формулы:

$$
\begin{gathered}
\psi_{0}(0)=\sqrt{1-\xi^{2}}, \\
\psi_{0}(t+1)=\xi \psi_{0}(t), \\
\psi_{k+1}(0)=-\xi \psi_{k}(0), \\
\psi_{k}(t+1)=\xi \psi_{k}(t)+\left(1-\xi^{2}\right) \sum_{i=0}^{k-1}(-\xi)^{k-i-1} \psi_{i}(t), \\
\psi_{k}(t)=(-1)^{k-t} \psi_{t}(k) .
\end{gathered}
$$

На рис. 1 изображены многочлены $\psi_{k}(t)$ при $\xi=0,7$.

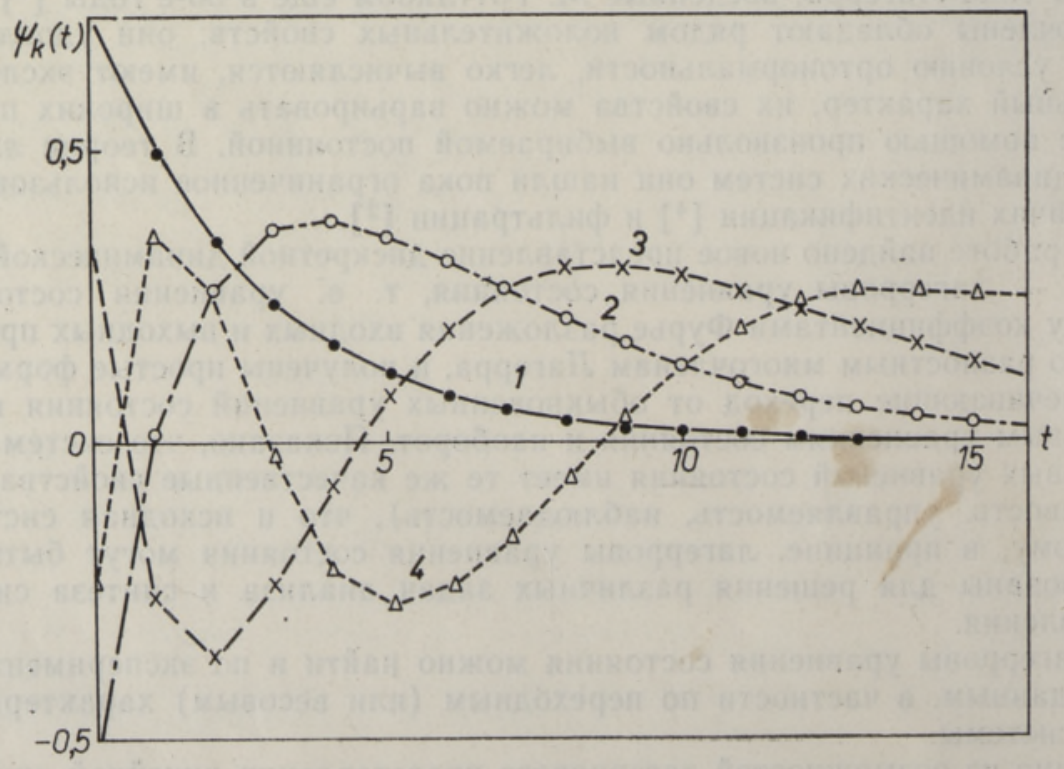

Рис. 1. Разностные многочлены Лагерра $\psi_{k}(t)$ при $\xi=0,7$ : $k=0(1), 1(2), 2$ (3) и $3(4)$. 
Так как многочлены $\psi_{k}(t)$ удовлетворяют условиям ортонормальности

$$
\sum_{t=0}^{\infty} \psi_{k}(t) \psi_{m}(t)=\delta_{k m},
$$

где $\delta_{k m}-$ символ Кронекера, то разложение дискретной функции $f(t)$, $t=0,1,2, \ldots$, в ряд Фурье по многочленам $\psi_{k}(t)$ имеет простой вид

$$
f(t)=\sum_{k=0}^{\infty} f_{k} \psi_{k}(t)
$$

где коэффициенты Фурье $f_{k}$ определяются соотношением

$$
f_{k}=\sum_{t=0}^{\infty} f(t) \psi_{k}(t)
$$

\section{3. Вывод лагерровых уравнений состояния}

Рассмотрим дискретную многомерную динамическую систему

$$
\begin{aligned}
& x(t+1)=F x(t)+G u(t), \\
& y(t)=H x(t), \quad t=0,1,2, \ldots,
\end{aligned}
$$

где $x-n$-вектор состояния, $u-m$-вектор управляющих воздействий, $y-p$-вектор выходных переменных системы. Матрицы $F, G, H$ имеют размерность $n \times n, n \times m$ и $p \times n$ соответственно.

Умножая обе стороны уравнений $(7)$ и $(8)$ на $\psi_{k}(t)$ и суммируя по $t=0,1,2, \ldots$, получим соотношения между векторами коэффициентов разложения дискретных функций $x(t), u(t)$ и $y(t), t=0,1,2, \ldots$, по разностным многочленам Лагерра

$$
\begin{aligned}
& \bar{x}_{k}=F x_{k}+G u_{k}, \\
& y_{k}=H x_{k}, \quad k=0,1,2, \ldots,
\end{aligned}
$$

где

$$
\bar{x}_{k}=\sum_{t=0}^{\infty} x(t+1) \psi_{k}(t) .
$$

На основании формул (3) и (5) получим следующее выражение для коэффициентов $\bar{x}_{k}$ :

$$
\begin{gathered}
\bar{x}_{k}=\xi \sum_{j=0}^{\infty} x_{j} \sum_{t=0}^{\infty} \psi_{j}(t) \psi_{k}(t)+ \\
+\left(1-\xi^{2}\right) \sum_{j=0}^{\infty} x_{j} \sum_{i=0}^{j-1}(-\xi)^{j-i-1} \sum_{t=0}^{\infty} \psi_{i}(t) \psi_{k}(t),
\end{gathered}
$$

а ввиду ортонормальности многочленов $\psi_{k}(t)-$

$$
\bar{x}_{k}=\xi x_{k}+\left(1-\xi^{2}\right) \sum_{i=1}^{\infty}(-\xi)^{i-1} x_{k+i} .
$$

Более подходящим соотношением между коэффициентами $\bar{x}_{k}$ и $x_{k}$, вытекающим непосредственно из формулы (11), является следуюшее:

$$
\bar{x}_{k}+\xi \bar{x}_{k+1}=\xi x_{k}+x_{k+1} \text {. }
$$


Вернемся теперь вновь к уравнениям (9) и (10). На основании (9) и (12) имеем

$$
x_{k+1}-\xi F x_{k+1}-\xi G u_{k+1}=F x_{k}+G u_{k}-\xi x_{k} .
$$

Обозначив

$$
z_{k}=(I-\xi F) x_{k}-\xi G u_{k},
$$

где $I$ - единичная матрица, из соотношений (11) и (8) получим

$$
\begin{aligned}
& z_{k+1}=A z_{k}+B u_{k}, \\
& y_{k}=C z_{k}+D u_{k},
\end{aligned}
$$

где матрицы

$$
\begin{aligned}
& A=(F-\xi I)(I-\xi F)^{-1}, \\
& B=\left(1-\xi^{2}\right)(I-\xi F)^{-1} G, \\
& C=H(I-\xi F)^{-1}, \\
& D=H(I-\xi F)^{-1} G .
\end{aligned}
$$

Назовем уравнения (15) и (16) лагерровыми уравнениями состояния системы (7)-(8), так как они представляют собой соотношения между коэффициентами $u_{k}, y_{k}$ разложения входных и выходных переменных по разностным многочленам Лагерра $\psi_{k}(t)$ в виде уравнений состояния. Используя формулы (17)-(20), легко перейти от исходной системы (7) - (8) к лагерровой системе (15)-(16) и наоборот:

$$
\begin{aligned}
& F=(I+\xi A)^{-1}(\xi I+A), \\
& G=(I+\xi A)^{-1} B, \\
& H=C(I-\xi F) .
\end{aligned}
$$

Рассмотрим теперь начальное состояние лагерровой системы $z_{0}$. При $k=0$ получим из (14)

$$
z_{0}=(I-\xi F) x_{0}-\xi G u_{0},
$$

а на основании (5) и (7) -

$$
z_{0}=\sum_{t=0}^{\infty}[x(t)-\xi x(t+1)] \psi_{0}(t) .
$$

Учитывая еще свойства (1) и (2) многочленов $\psi_{0}(t)$, получим для определения начального состояния лагерровой системы следующее выражение

$$
z_{0}=\sqrt{1-\xi^{2}} x(0)
$$

Формула (24) имеет большое практическое значение. В задачах управления и идентификации часто предполагается $x(0)=0$. Тогда и $z_{0}=0$. Если $\xi \rightarrow 1$, то при любом $x(0),|x(0)|<\infty$ имеет место $z_{0} \rightarrow 0$. А при $\xi \rightarrow 0$ верно $z_{0} \rightarrow x(0)$.

Интересно отметить, что при $\xi \rightarrow 0$ лагеррова система $(15)-(16)$ приближается к исходной системе $(7)-(8)$, а при $\xi \rightarrow 1$ на основании формул $(17)-(20)$ имеем

$$
\lim _{\zeta \rightarrow 1} y_{k}=H(I-F)^{-1} G u_{k} .
$$




\section{4. Устойчивость лагерровой системы}

Пусть система (7)-(8) асимптотически устойчива, т. е. максимальное собственное число матрицы $F$ по модулю меньше единицы. Рассмотрим характеристический многочлен матрицы $A$

$$
\begin{aligned}
\operatorname{det}(\lambda I-A) & =\operatorname{det}\left[\lambda I-(F-\xi I)(I-\xi F)^{-1}\right]= \\
& =\operatorname{det}[(\lambda+\xi) I-(1+\xi \lambda) F] \operatorname{det}(I-\xi F)^{-1} .
\end{aligned}
$$

Предположим, что $|\lambda|<1$. Тогда $1+\xi \lambda \neq 0$, так как $|\xi|<1$. Для устойчивой $F$ получим теперь

$$
\operatorname{det}(\lambda I-A)=0,
$$

если только

$$
\operatorname{det}((\lambda+\xi) /(1+\xi \lambda) I-F)=0 .
$$

Значит, характеристические числа $\lambda$ и $\bar{\lambda}$ матриц $A$ и $F$ соответственно связаны формулой

$$
\lambda=(\bar{\lambda}-\xi) /(1-\xi \bar{\lambda}) \text {. }
$$

Пусть

$$
\bar{\lambda}=|\bar{\lambda}|(\cos \varphi+i \sin \varphi)
$$

Тогда

$$
\begin{aligned}
|\lambda|^{2} & =\left(|\bar{\lambda}|^{2}-2 \xi|\bar{\lambda}| \cos \varphi+\xi^{2}\right) /\left(1-2 \xi|\bar{\lambda}| \cos \varphi+\xi^{2}|\bar{\lambda}|^{2}\right)= \\
& =Q(\bar{\lambda}, \xi) / P(\bar{\lambda}, \xi) .
\end{aligned}
$$

Очевидно, $Q(\bar{\lambda}, \xi) \geqslant 0$ и $P(\bar{\lambda}, \xi) \geqslant 0$, так как при наихудшей $\varphi$

$$
\begin{aligned}
& Q(\bar{\lambda}, \xi)=(|\bar{\lambda}| \pm \xi)^{2}, \\
& P(\bar{\lambda}, \xi)=(1 \pm \xi|\bar{\lambda}|)^{2} .
\end{aligned}
$$

Пусть $|\bar{\lambda}|<1$. Тогда и $|\lambda|<1$, так как

$$
P(\bar{\lambda}, \xi)-Q(\bar{\lambda}, \xi)=\left(1-\xi^{2}\right)\left(1-|\bar{\lambda}|^{2}\right)>0
$$

и $|\xi|<1$. Следовательно, лагеррова система будет асимптотически устойчивой, если только исходная система асимптотически устойчива. Значит, сделанное нами предположение, а также формула (25) выполняются для асимптотически устойчивых систем. Из формулы (25) вытекают следующие интересные частные случаи: если $\bar{\lambda}=0$, то $\lambda=-\xi ;$ если $\bar{\lambda}=\xi$, то $\lambda=0$; если $\xi \rightarrow 0$, то $\lambda \rightarrow \bar{\lambda}$; если $\xi \rightarrow \pm 1$, то $\lambda \rightarrow \mp 1$.

\section{5. Управляемость и наблюдаемость лагерровой системы}

Воспользуемся следующими критериями управляемости и наблюдаемости $\left[{ }^{5}\right]$ :

- пара $(A, B)$ полностью управляема, если

$$
\operatorname{rank}\left[\lambda_{i} I-A \vdots B\right]=n, \quad i=1, \ldots, n,
$$

- пара $(A, C)$ полностью наблюдаема, если 


$$
\operatorname{rank}\left[\underline{\lambda_{i} I-A}-\frac{A}{C}\right]=n, \quad i=1, \ldots, n
$$

Покажем теперь, что

$$
\operatorname{rank}\left[\lambda_{i} I-A \vdots B\right]=\operatorname{rank}\left[\bar{\lambda}_{i} I-F \vdots G\right] .
$$

На основании (17) и (18) имеем, учитывая еще перестановочность матриц $F-\xi I$ и $I-\xi F$,

$$
\operatorname{rank}\left[\lambda_{i} I-A \vdots B\right]=\operatorname{rank}\left[\frac{\xi+\lambda_{i}}{1+\xi \lambda_{i}} I-F: \frac{1-\xi^{2}}{1+\xi \lambda_{i}} G\right] \text {, }
$$

откуда непосредственно следует (26), так как $|\xi|<1$.

На основании (17) и (19) аналогично получаем

$$
\operatorname{rank}\left[--\frac{\lambda_{i} I-A}{C}\right]=\operatorname{rank}\left[\begin{array}{c}
\frac{\xi+\lambda_{i}}{1+\xi \lambda_{i}} I-F \\
\frac{1}{1+\xi \lambda_{i}} H
\end{array}\right] \text {, }
$$

а учитывая еще $(25)$, -

$$
\operatorname{rank}\left[-\frac{\lambda_{i} I-A}{C}-\frac{A}{C}\right]=\operatorname{rank}\left[\frac{\bar{\lambda}_{i} I-F}{\bar{H}}--\right]
$$

Значит, лагеррова система $(A, B, C)$ полностью управляема и наблюдаема, если только исходная система $(F, G, H)$ полностью управляема и наблюдаема.

\section{6. Определение лагерровых уравнений состояния по матрице переходных функций}

Пусть задана матрица переходных функций системы

$$
S(t)=\left[s_{\alpha \beta}(t)\right], \quad \alpha=1, \ldots, p, \quad \beta=1, \ldots, m, \quad t=0,1,2, \ldots,
$$

где

$$
s_{\alpha \beta}(t)=\left[y_{\alpha}(t) \mid x(0)=0, u_{\beta}(\tau)=1, \tau=0, \ldots, t\right] .
$$

По формуле (6) найдем лагеррово разложение переходной функции $s_{\alpha \beta}(t)$ (индексы $\alpha$ и $\beta$ в дальнейшем опускаются)

$$
s_{k}=\sum_{t=0}^{\infty} s(t) \psi_{k}(t), \quad k=0,1,2, \ldots .
$$

Коэффициенты $s_{k}$ можно определить и как реакцию лагерровой модели (15) - (16) на входное воздействие $u_{i}, i=0, \ldots, k$,

$$
s_{k}=\sum_{i=0}^{k} m_{i} u_{i}
$$

где $m_{i}$ - марковские параметры лагерровой модели

$$
\begin{aligned}
& m_{i}=c^{\prime} A^{i-1} b, \quad i=1,2, \ldots, \\
& m_{0}=d,
\end{aligned}
$$


a $u_{i}$ - разложение входного сигнала $u(t)$ по разностным многочленам Лагерра.

Для единичного входного сигнала $u(t)=1, t=0,1, \ldots$, имеем

$$
u_{i}=\sum_{t=0}^{\infty} \psi_{i}(t)
$$

Покажем, что

$$
\sum_{t=0}^{\infty} \psi_{i}(t)=\sigma=\mathrm{const}, \quad i=0,1,2, \ldots
$$

На основании свойств симметрии (4) и смещения (3) разностных многочленов Лагерра имеем

$$
\begin{gathered}
\sum_{t=0}^{\infty} \psi_{i+1}(t)=\sum_{t=0}^{\infty}(-1)^{i-t+1} \psi_{t}(i+1)= \\
=\xi \sum_{t=0}^{\infty}(-1)^{i-t+1} \psi_{t}(i)+\left(1-\xi^{2}\right) \sum_{t=0}^{\infty} \sum_{j=0}^{t-1}(-1)^{i-j \xi^{t-j-1} \psi_{j}(i) .}
\end{gathered}
$$

Изменив порядок суммирования во втором слагаемом, получим

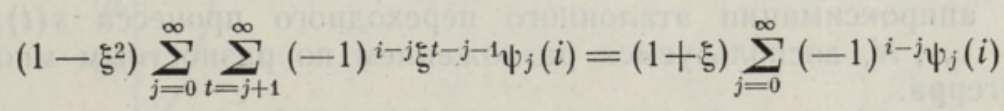

и в итоге

$$
\sum_{t=0}^{\infty} \psi_{i+1}(t)=\sum_{t=0}^{\infty}(-1)^{i-t} \psi_{t}(i)=\sum_{t=0}^{\infty} \psi_{i}(t) .
$$

Следовательно, утверждение (30) выполнено и поэтому с учетом (1) и (2) имеем

$$
\psi_{0}(t)=\xi^{t} \sqrt{1-\xi^{2}}
$$

и

$$
\sigma=\sum_{t=0}^{\infty} \psi_{0}(t)=\sqrt{(1+\xi) /(1-\xi)} .
$$

На основании (28)-(30) получаем следующие простые выражения между марковскими параметрами лагерровой модели $m_{i}$ и коэффициентами разложения $s_{i}$ :

$$
m_{i}=\left(s_{i}-s_{i-1}\right) / \sigma, \quad i=1,2, \ldots .
$$

Определяя из соотношения (31) марковские параметры $m_{i}(\alpha \beta)$ между всеми входами и выходами, составляем $(p \times m)$-матрицы марковских параметров $M_{i}, i=0,1,2, \ldots$, лагерровой модели многомерной системы

$$
M_{i}=\left[m_{i}(\alpha \beta)\right], \quad \alpha=1, \ldots, p, \quad \beta=1, \ldots, m
$$

Очевидно,

$$
D=M_{0},
$$

а матрицы $A, B$ и $C$ лагерровых уравнений состояния определяются по матрицам $M_{i}, i=1,2, \ldots$, обычными методами реализации $\left[{ }^{6}\right]$.

Отметим еще, что марковские параметры лагерровой модели $m_{i}$ можно легко определить по весовой функции $h(t)$ системы 


$$
h(t)=[y(t) \mid x(0)=0 ; u(0)=1 ; u(t)=0, t \neq 0] .
$$

Тогда

a

$$
u_{i}=\psi_{i}(0) \text {, }
$$

$$
m_{i}=\psi_{0}^{-1}(0)\left(h_{i}+\xi h_{i-1}\right),
$$

где $h_{i}$ - лагеррово разложение весовой функции $h(t)$.

\section{7. Определение эталонной модели по показателям качества}

Традиционные показатели качества управления, такие как время регулирования, максимальное отклонение регулируемой величины от установившегося значения, колебательность, определяют некоторые существенные значения желаемого переходного процесса.

Задача синтеза многомерной системы решается относительно просто и изящно, если желаемая динамика замкнутой системы задана в виде уравнений в переменных состояния. Отсюда возникает важная задача построение уравнений в переменных состояния по традиционным показателям качества управления.

Для аппроксимации эталонного переходного процесса $s(t), t=$ $=0,1, \ldots, N$, воспользуемся разложением по разностным многочленам Лагерра.

C точки зрения проектировщика системы управления не все значения функции $s(t)$ одинаково существенны. Поэтому при построении эталонной модели введем весовые коэффициенты $w(t)$ с тем, чтобы наиболее существенным значениям придать больший вес. ловия

Коэффициенты разложения $s_{k}, k=0,1, \ldots, M$, определяем из ус-

$$
\min _{s_{0} \ldots s_{\mathbf{M}}} \sum_{t=0}^{N} w(t)\left[s(t)-\sum_{k=0}^{M} s_{k} \psi_{k}(t)\right]^{2},
$$

где $N>M$.

Введем следующие обозначения

$$
\begin{gathered}
\Psi=\left[\begin{array}{ccc}
\psi_{0}(0) & \ldots & \psi_{M}(0) \\
\psi_{0}(N) & \ldots & \psi_{M}(N)
\end{array}\right], \\
p^{\prime}=\left(s_{0} \ldots s_{M}\right), \quad q^{\prime}=(s(0) \ldots s(N)), \\
W=\operatorname{diag}(w(0) \ldots w(N)) .
\end{gathered}
$$

Теперь соотношение (32) можно переписать в виде

$$
\min _{p}(q-\Psi p)^{\prime} W(q-\Psi p) .
$$

Хорошо известно, что решением (33) является

$$
p=\left(\Psi^{\prime} W \Psi\right)^{-1} \Psi W q .
$$

Отметим, что в силу ортонормальности многочленов $\psi_{k}(t)$ матрица $\Psi^{\prime} W \Psi$ хорошо обусловлена и поэтому вычисление вектора коэффициентов $p$ всегда осуществимо.

Схема построения эталонной модели: 
1. По заданным показателям качества определяем опорные точки $s\left(t_{i}\right)$ желаемого переходного процесса.

2. Проведя по опорным точкам кусочно-линейную аппроксимацию, получаем вспомогательные точки желаемого переходного процесса.

3. Задаем отношение весовых коэффициентов опорных и вспомогательных точек.

4. Задаем общее число $M$ и постоянную $\xi$ аппроксимирующих многочленов $\psi_{k}(t)$.

5. По формуле (34) определяем коэффициенты разложения $\boldsymbol{s}_{k}$.

6. Вычисляем марковские параметры $m_{i}$ по формуле (31).

7. Составляем матрицы марковских параметров $M_{i}$.

8. Методом канонической реализации находим лагерровы уравнения состояния (15)-(16).

9. Параметры эталонной модели определяем по формулам (21) - (23).

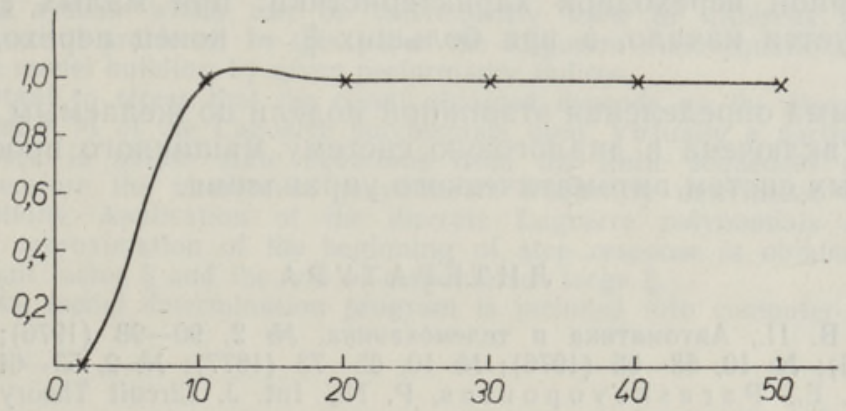

Рис. 2. Эталонный переходный процесс.

На рис. 2 показаны результаты построения эталонной модели согласно описанной выше процедуре. Опорные точки желаемого переходного процесса изображены крестиками. Параметры эталонной модели, вычисленные по заданным исходным данным, при отношении весовых коэффициентов $w=5$ и постоянной Лагерра $\xi=0,8$ следующие:

$$
\begin{aligned}
& F=\left[\begin{array}{ccr}
0,746 & 0,0815 & -0,679 \\
0,0679 & 1,150 & 0,848 \\
-0,0849 & -0,435 & 0,190
\end{array}\right], \quad G=\left[\begin{array}{r}
0,335 \\
0,405 \\
-0,204
\end{array}\right], \\
& H=[0,403-0,06520,543] .
\end{aligned}
$$

\section{8. Заключение}

В работе выведены уравнения состояния для коэффициентов разложения входных и выходных переменных многомерной дискретной динамической системы по разностным многочленам Лагерра. При этом получены простые формулы, обеспечивающие переход от исходной системы к лагерровой и наоборот. Качественный анализ показал, что лагеррова система устойчива, управляема и/или наблюдаема, если только исходная система устойчива, управляема и/или наблюдаема соответственно. Поэтому лагерровы уравнения состояния являются полноценной моделью представления динамической системы.

Отметим, что лагерровы уравнения состояния приобретают практическую ценность при преобразовании конечных последовательностей 
При этом выявляются сглаживающие и сжимающие свойства преобразования Лагерра, которые можно в широких пределах варьировать изменением постоянной ६. Лагерровы уравнения состояния представляют собой в таких случаях некоторую аппроксимацию исходной системы, которую удобно использовать при различных задачах идентификации, фильтрации и управления.

Отмеченные положительные свойства лагеррового представления системы нашли использование при построении эталонной модели системы управления по заданным показателям качества. При этом следует отметить, что получаемый результат зависит в первую очередь от величины лагерровой постоянной $\xi$ и длины лагерровой последовательности $M$. Число $M$ определяет порядок получаемых уравнений состояния, так как в сущности решается задача частичной реализации. Постоян-

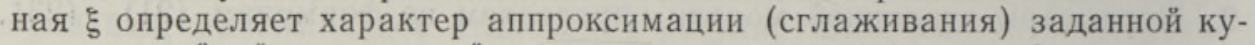
сочно-линейной переходной характеристики: при малых $\xi$ лучше аппроксимируется начало, а при больших $\xi-$ конец переходной характеристики.

Программа определения эталонной модели по желаемым переходным процессам включена в диалоговую систему машинного проектирования многомерных систем автоматического управления.

\section{Л И ТЕ РА Т У РА}

1. Перов В. П., Автоматика и телемеханика, № 2, 90-98 (1976); № 8, 81-86 (1976); № 10, 58-65 (1976); № 10, 65-73 (1977); № 2, 53-61 (1978).

2. King, R. E., Paraskevopoulos, P. N., Int. J. Circuit Theory and Appl., 5, № 1, 81-91 (1977).

3. Gottli eb, M., Amer. J. Math., 60, 453-458 (1938).

4. Paraskevopoulos, P. N., King, R. E., In: Identification and System Parameter Estimation, Part 2, IV IFAC Symposium, Moscow, 1976, p. 536-543.

5. Davison, E. J., Wang, H. S., IEEE Trans. Automat. Contr., AC-20, № 1, $123-128(1975)$.

6. Silverma n, L. M., IEEE Trans. Automat. Contr., 16, № 6, 554-567 (1971).

Институт кибернетики

Академии наук Эстонской ССР
Поступила в редакцию $26 /$ II 1981

\section{U. NURGES, O. JAAKSOO}

\section{MITMEMOOOTMELISE DISKREETSE SUSTEEMI LAGUERRE'I OLEKUMUDEL}

On leitud lineaarse diskreetse dünaamilise süsteemi Laguerre’i olekumudel, s.t. olekuvōrrandid süsteemi sisend- ja väljundsuuruste diskreetsete Laguerre'i reaksarenduste vahel. On saadud lihtsad valemid üleminekuks tavaliselt olekumudelilt Laguerre'i olekumudelile ja vastupidi ning näidatud, et stabiilse, juhitava $\mathrm{ja} /$ või jälgitava süsteemi Laguerre'i olekkumudel on stabiilne, juhitav ja/või jälgitav. Laguerre'i olekumudelit on kasutatud soovitud etalonmudeli leidmiseks etteantud kvaliteedinäitajate järgi. 


\section{LAGUERRE'S STATE EQUATIONS FOR MULTIVARIABLE DISCRETE-TIME SYSTEMS}

In this paper the state equations for the expansion coefficients of input and output variables of a multivariable discrete time dynamical system by the discrete Laguerre polynomials are derived. Simple formulas for the transformation of the original state equations to the Laguerre (17) - (20) and vice versa (21) - (23) are obtained. Qualitative analysis revealed that Laguerre system is stable, controllable and/or observable if only the original system is stable, controllable and/or observable. Thus the Laguerre state equations are of a full value representation of a dynamical system.

It is worth emphasising that the Laguerre state equations acquire practical value for transformation of finite input-output sequences. Then smoothing properties of the Laguerre transformation occur which can be varied within a great extent by changing the discount factor $\xi$. The Laguerre state equations then represent an approximation of the original system which can be conveniently used in different identification, filtration and control problems. In this paper the Laguerre state equations have found use in reference model building by given performance indices.

It is important to stress that the result obtained depends on the discount factor $\xi$ and on the number $M$ of the Laguerre polynomials used. Virtually a partial realization problem is solved to obtain state equations from the finite sequences of expansion coefficients. Therefore the number of polynomials frequently determines the order of the state equations. Application of the discrete Laguerre polynomials demonstrates that the better approximation of the beginning of step response is obtained for small values of discount factor $\xi$ and the end of response for large $\xi$. system.

The reference model determination program is included into computer-aided design 